\title{
Epidemiologia e planejamento: a recomposição das práticas epidemiológicas na gestão do SUS
}

\author{
Epidemiology and planning: the recomposition of \\ the epidemiological practices in management of \\ SUS
}

Abstract This paper has the aims of synthesizing some of the approaches related with the use of epidemiology in health services, of describing some proposals and of discussing the feasibility and obstacles for structuring epidemiological practices inside Unified Brazilian H ealth System (SUS). The essay describes some aspects of the epidemiology's crisis and analysis some constraints to the use of epidemiological knowledge in health services management. The main achievements and turn backs related to that issue, during the SU S implementation processes, areidentified. Finally, some proposals related to the development of a counter hegemonic epidemiology are discussed looking forward the creation of a new public health practice based on planning and management of a effective, democratic, human and equitable health system.

Key words Epidemiology, $\mathrm{H}$ ealth services, Planning
Resumo Com os objetivos de sistematizar os esforços para a utilização da epidemiologia nos servi ços de saúde, descrever algumas propostas construídas no Brasil e discutir obstáculos e possibilidades de recomposi ção das práticas epidemiológicas no Si stema Ú nico de Saúde (SUS), o ensaio apresenta elementos da crise da epidemiologia e analisa certos constrangimentos impostos ao desenvolvimento da racionalidade técnico-sanitária e à incorporação tecn ológica do saber epidemiológico na gestão em saúde. São identificados a van ços e recuos desses processos durante a implementação do SU S e apresentadas al gumas proposições para a construção col etiva de uma epidemiologia contra-hegemônica que contribua na constituição de suj eitos sociais comprometidos com uma prática sanitária que aposte na planificação e gestão de um sistema de saúde efetivo, democrático, humanizado e equânime.

Palavras-chave Planejamento e gestão em saúde, Prática epidemiológica, Epidemiologia em serviços de saúde
1 Instituto de Saúde Coletiva da U niversidade Federal da Bahia. Rua Padre Feijó 29, 40 andar. Campus Canela 40210-070, Salvador BA. jairnil@canudos.ufba.br 


\section{Introdução}

Há quase meio século tem-se apontado para os usos da epidemiologia na descrição da doença na comunidade, na identificação de grupos vulneráveis e na avaliação de serviços e programas de saúde ( M orris, 1975). No entanto, a constituição da epi demi ologia como disciplina científica e a reflexão epistemológica sobre a mesma segui ram, por al gum tempo, os seus próprios caminhos, sem um vínculo mais consistente com a organização social dos serviços de saúde, dada a relativa autonomia dos campos científicos.

A ênfase nos estudos etiológicos, no desenvolvimento metodológico e mesmo na formalização da disciplina (Barata, 1998) distanciava, de certo modo, muitos dos epidemiologistas de um pensamento e ação sobre os serviços de saúde. A hegemonia das universidades norte-american as e dos centros de epidemiologia dos Estados U nidos, a exemplo dos Centers for Disease Control and Prevention (CDC, 1992), na formação de epidemi ologistas do mundo inteiro e, particularmente, dos países dependentes, reforçava tal situação. Os serviços de saúde, por sua vez, muito mais orientados sob a lógica do mercado do que a das necessidades de saúde pareciam não ver a epidemiologia como uma ferramenta necessária para o seu desenvolvimento.

Evidentemente que muitos países do "socialismo real" e aqueles capitalistas que realizaram profundas modificações na organização dos serviços de saúde - como o Reino Unido, com a implantação do $\mathrm{N}$ ational $\mathrm{H}$ ealth Services a partir de 1948, e o Canadá, desde o Relatório Lalonde em 1974 - exploraram de forma mais ampla as potencial idades científicas e tecnológicas da epidemiologia na gestão de sistemas de serviços de saúde (Paim, 2002).

A identificação desse gap entre as possibilidades das práticas epidemiológicas e a sua utilização pelos serviços da saúde para além do controle de doenças transmissíveis ou da vigilância epidemiológica esti mulou a O rganização Pan-Americana da Saúde e a Organização M undial da Saúde (OPS/OM S) a apoiarem um conjunto de iniciativas visando à incorporação e à utilização desse saber no âmbito dos sistemas de saú de (OPS, 1984; 1988; 1991).

0 presente artigo tem como objetivos sistematizar os esforços visando à utilização da epidemiologia pelos serviços de saúde na
América Latina nas últimas décadas, descrever al gumas das propostas construídas no Brasil e discutir certos obstáculos e possibilidades de recomposição das práticas epidemiológicas na gestão do Sistema ú nico de Saúde(SUS).

\section{0 papel da epidemiologia nos serviços e sistemas de saúde}

N as duas últimas décadas a O rganização PanAmericana de Saúde tem demonstrado uma preocupação com processos de mudança nos procedimentos técnicos de prestação dos serviços, no uso das tecnologias disponíveis, na integração dos conhecimentos, nas formas de utilização dos recursos e nos modos de concretizar a participação social (OPS, 1984; 1988; 1991; Tigre et al., 1990).

Ao propugnar por ações integradas de saúde, esse organismo internacional ratificava o princípio da integralidade adotado pelo movimento sanitário brasileiro desde a $8 \underline{a}$ Conferência Nacional de Saúde e incluído como diretriz no capítulo saúde da Constituiçãao de 1988. Recomendava, assim, um enfoque global das ações agrupadas de acordo com o conjunto de problemas da população, sendo executadas de forma integral, evitando-se os agrupamentos por patologias e programas isolados. Ademais, defendia um serviço de saúde organizado para produzir mudança no perfil epidemiológico com relação aos problemas de saúde (riscos edanos), coletivos eindividuais.

Parte dessa discussão se expressou no desenvolvimento da proposta de Sistemas Locais de Saúde (Silos) e na reflexão e crítica aos model os de prestação de serviços de saúde ou model os de aten ção possibilitadas pela concepção e implantação dos distritos sanitários, como modos de reorganização das práticas de saúde (Paim, 1993).

Ao se discutir o uso da epidemiologia nos sistemas e serviços de saúde, é comum ressaltar o papel da disciplina na produção de conhecimentos para a tomada de decisões no que se refere à formulação de políticas de saúde, à organização do sistema eàs intervenções destinadas a dar solução a problemas específicos (Tigre et al., 1990). N esse particular, identificam-se os seguintes cam pos de ação para a disciplina no âmbito dos serviços de saúde: a) estudos da situação de saúde em di- 
ferentes grupos da população, seus determinantes e tendências; b) vigilância epidemiológica de doenças e de outros problemas de saúde; c) investigação causal e explicativa sobre problemas prioritários de saúde; d) avaliação do impacto em saúde dos serviços, de tecnologias e de outras ações.

Refletindo sobre os processos de tomada de decisões em saúde e, particularmente, sobre a aproximação entre epidemiologia e gestão, Dussault (1995) enumera as seguintes possibilidades de utilização: a) nas políticas públicas de saúde, apoiando a definição de prioridades, objetivos e estratégias; b) na configuração dos serviços, especialmente na descentralização e integração dos serviços nos programas; c) nas práticas dos profissionais, sobretudo na avaliação da eficiência e eficácia; d) nas práticas de gestão; e) nas prioridades de investigação.

A pós extensa revisão da literatura sobre limites e possibilidades do "enfoque epidemiológico", Teixeira (1996) destaca o grande dinamismo da produção científica da área no Brasil e a contribuição da epidemiologia ao desenvolvimento teórico-metodológico do planejamento de saúde. Considera que a reorientação da gestão, do financiamento, da organização e do modelo assistencial do sistema de serviços de saúde constituem processos que não podem prescindir da epidemiologia, enquanto saber científico e prática instrumental que confere especificidade aos objetos de conhecimento e de interven ção no âmbito da saúde em sua dimensão populacional, isto é coleti va (Teixeira, 1999). N esse particular, enumera os seguintes usos da epidemiologia: a) no processo de formulação de políticas; b) na definição de critérios para a repartição de recursos; c) na elaboração de diagnósticos e análises de situação de saú de; d) na elaboração de planos e programas; e) na organização de ações e serviços; f) na avaliação de sistemas, políticas, programas e serviços de saúde. Ao discutir os limites e as possibilidades de desenvolvimento do "enfoque epidemiológico" no processo de reorientação da gestão eda organização social das práticas de saúde, a autora dedica uma reflexão especial para o papel da epidemiologia na própria definição do objeto do planejamento de saúde.

\section{Redefinições no estudo da gestão em saúde e tendências contemporâneas}

\section{da epidemiologia}

Para a discussão deste tópico faz-se necessário refletir sobre questões referentes à gestão de sistemas e serviços de saúde, evisitar, ainda que brevemente, al guns el ementos da chamada "crise da epidemiologia" e certas propostas de superação.

\section{Redefinições da gestão}

Embora sem uma consistente el aboração teórica, consubstanciada em investigações concretas, é freqüente vincular a epidemiologia à gestão como se fora al go natural e racional. Assim, prescrevem-se recomendações para a sua utilização no processo decisório como se a incorporação tecnológica pudesse ser efetivada num campo neutro ou inteiramente receptivo faceà racionalidade e aos benefícios desse saber.

No entanto, a gestão em saúde pode ser reconhecida como a direção ou condução de processos político-institucionais relacionados ao sistema de saúde, eminentemente conflituosos ou contraditórios, ain da que nos níveis técnico-administrati vo e técnico-operacional desenvolva ações de planejamento, organização, gerenciamento, controle e avaliação dos recursos humanos, financeiros e materiais empregados na produção de serviços de saúde. N essa perspectiva, a noção de gestão se aproxima da proposta de admini stração estratégica (OPS/OM S, 1992), na qual podem ser valorizados os componentes vinculados à distribuição dos diferentes tipos de poder em saúde e à construção de viabilidade das interven ções desenhadas.

U ma via de aproximação a essa temática desenvolvida no Brasil tem sido o recurso ao conceito de práticas de saúde, elaborado pioneiramente por Cecília Donnângelo e Ricardo Bruno $M$ endes-Gonçalves (Donnângelo, 1976; M en des-Gonçal ves, 1994). Essa via tende a produzir reflexões e análises so bre planificação e gestão que não se limitam a pensar objetos-meio (recursos financeiros, materiais, "informacionais", de poder, etc.), isoladamente, mas suas articulações com objetos-fim (interven ção, assistência, acolhimento, cuidado, etc.) em uma totalidade social.

Desse modo, a formulação da teoria do processo de trabalho em saúde possibilitou uma reflexão sobre o objeto, os meios de trabalho, as atividades e os agentes (agentes- su- 
jeitos ou sujeitos/agentes). Além disso, ao investigar as relações técnicas e sociais sob as quais se realiza o trabalho em saúde, o método histórico-estrutural permite o estudo das organizações, da distribuição do poder nas instituições edo processo decisório para a implementação de políticas ( Paim, 2002). Essa linha de estudos tem realizado, recentemente, abordagens mais processuais das organizações, trazendo a interatividade e a comunicação dos sujeitos como objetos de reflexão, deslocando a ênfase da administração das coisas e para a condução de processos político-institucionais e de trabalho em saúde. Converge, desse modo, com a gestão estratégica na qual a "administração da incerteza" centra-se na condução de processos coletivos de trabalho (M ota, 1992), val orizando a construção de consensos.

Assim, a gestão não se reduz à ação instrumental sobre o mundo objetivo (Rivera, 1995). Pode ser considerada analiticamente em uma dupla dimensão de ação social: ação estratégica dirigida a fins, com a criação de consenso ativo entre dirigentes e dirigidos, e ação comunicativa voltada para o entendimento e para as trocas intersubjetivas na constituição de novos sujeitos das práticas institucionais de saúde. N esse caso, cabe ressaltar a natureza ético-política da gestão em saúde, como resposta a necessidades humanas e, simultaneamente, formas de sociabilidade (Paim, 1999). Ao se examinarem os sujeitos em ação e em comunicação nas organizações, é possível analisar a interação social, identificar as racional idades presentes na gestão e valorizar a intersubjetividade na explicitação de conflitos e na busca de enten dimento. 0 saber epidemiológico, nessa perspectiva, comporia parte da racional idade técnicosanitária presente na explicação da situação de saúde (Teixeira, 1999) e compete, no processo decisório das instituições de saúde, com outras racionalidades igual mente importantes: política, médico-assistencial, econômica e burocrática (Paim, 2002).

É possível que o recurso aos conceitos de tecnologias leves, leves-duras e duras ( $M$ erhy, 1997) e a análise dessas racionalidades ajudem a compreender a incorporação da epidemiologia no planejamento e gestão em saúde. Assim, a partir de uma concepção de modelos assistenciais ou modos estruturados de intervenção em saúde que signifiquem combinações de tecnologias orientadas para 0 atendi- mento de necessidades, poder-se-ia investigar o uso da epidemiologia para a apreensão da dimensão coletiva dos problemas de saúde.

Essas indicações fazem supor a pertinência de estudos de incorporação tecnológica e de inovação ou desenvolvimento institucional para examinar a epidemiologia nos serviços de saú de. Ou seja, não basta o acúmulo de um saber, a existência de uma tecnologia nem o reconhecimento de uma necessidade social para que a epidemiologia seja utilizada por uma organização. Agentes capacitados, recurso ao planejamento, influência na gestão, liderança, etc. podem ser variáveis a serem examinadas na análise das possibilidades de incorporação. Conceitos outros, compondo quadros teóricos distintos, poderiam apontar outras variáveis a serem contempladas em investigações sobre políticas, instituições e práticas de saúde (Paim, 2002).

Ao se analisarem possíveis relações entre epidemiologia, planejamento e gestão caberia examinar modos de articular as dimensões políticas com a técnico-científica nas intervenções em saúde, tal como se propõe na discussão dos model os assistenciais (Paim, 1993; 2002). Além de uma "tecnologia de poder" ou de uma técnica que ajuda a dispor, arranjar e processar outras técnicas, bem como organizar e dirigir processos de trabal ho, o planejamento pode ser um meio de auxiliar a interação entre os sujeitos no sentido de viabilizar um dado projeto ético-político para a saúde (Paim, 1999).

\section{Tendências da epidemiologia}

$\mathrm{N} o$ que se refere à epidemiologia faz-se necessário concebê-la, para fins de análise da sua utilização na gestão, como disciplina científica e como meio de trabalho.

$\mathrm{N}$ a primeira acepção trata-se de pensar 0 saber científico e os paradigmas em que se insere a ciência epidemiológica, o que remete para uma trabal ho teórico e para uma reflexão epistemológica. Assim, caberia problematizar a crise do paradigma dominante, a capacidade de formulação teórica, a ruptura dos compromissos históricos, a relação com a práxis e a capacidade explicativa (Barreto, 1998). Segundo esse autor, a "crise da epidemiologia" no que diz respeito ao desgaste da sua capacidade explicativa se expressa nas seguintes situações: a) as propostas de prevenção fator a fator são de difícil implementação 
e apresentam uma "eficiência" limitada; b) a avaliação de tecnologias não oferece um quadro completo dos efeitos previstos e imprevistos quando são utilizadas como parte de programas complexos de intervenção em saúde; c) a capacidade de previsão dos efeitos dos programas e ações geral mente é baixa, independentemente das boas intenções e da consistência dos conhecimentos disponíveis; d) parecem esgotadas as possibilidades de gerar novos con hecimentos acerca de fatores de risco com forças associativas el evadas ou com alto grau de especificidade em relação aos seus efeitos.

Consequentemente, mesmo epidemiologistas comprometidos com a melhoria dos serviços de saúde, como o referido autor, ainda são reticentes quanto às possibilidades da disciplina no interior do sistema de serviços de saúde. Todavia, a pujança dos vários congressos brasi leiros de epidemiologia e de saúde coletiva no Brasil parece indicar o contrário, quando se destacam as suas contribuições no estudo das desigualdades, na planificação, gestão e avaliação das intervenções em saúde (Teixeira, 1996).

Pensar a epidemiologia como meio de trabalho significa concebê-la como tecnologia, ou seja, ferramenta de gestão. Assim, a epidemiologia, como saber tecnológico, pode ser investigada na sua aplicação como instrumento para a formulação de políticas, para a planificação e para avaliação em saúde (Schraiber et al., 1999). N essa perspectiva, poder-se-ia examinar a sua utilização na análise da situação de saúde (investigando o modo e as condições de vida dos grupos sociais que se inserem e se movimentam no espaço urbano), no desenvolvimento de tecnologias, na elaboração e teste de model os assistenciais. 0 saber epidemiológico, como tecnologia não material, poderia ser utilizado na organização de processos de trabalho, de serviços e de sistemas de saúde, bem como na planificação, gestão, vigilância e avaliação em saúde (Paim, 1999).

\section{Epidemiologia e gestão: alguns desafios}

$\mathrm{N}$ a perspectiva da saúde coletiva, entre os desafios da epidemi ologia estão o estudo das desigual dades em saúde; o desenvolvimento de um pensamento sobre ambiente, qualidade de vida, conceito e medidas de saúde; a pesquisa sobre avaliação, seleção de tecnologiase intervenções em saúde (Barreto, 1998).

No âmbito do planejamento e da gestão, caberia retomar propostas de diagnósticos administrativo, estratégico e ideológico (Testa, 1992), assumindo o poder como categoria central de análise e identificando como seus objetos os serviços, organizações e sistemas, além de necessidades/problemas de saúde (Teixeira, 1999). Esse obj eto de trabal ho poderia ser delimitado, portanto como a relação entre os problemas de saúde e as respostas sociais aos mesmos (Sá, 1993), o que significa pensar o planejamento e a gestão na sua articulação com as instâncias política, econômica eideológica que compõem a estrutura social. Eis, portanto, um dos grandes desafios para o planejamento e a gestão em saúde:

Articular em seu interior como prática social, tanto a explicação dos problemas de saúde dos distintos grupos populacionais na perspectiva apontada pela "epidemiologia crítica", enfatizando as relações entre os problemas de saúde, as condições de vida e seus determinantes histórico-estruturais, quanto a compreensão das representações sociais acerca da saú de-doença e atenção à saúde dos diversos grupos, o que indica a necessidade de um planejamento participativo, em que os especialistas e população sejam atores e autores das respostas sociais aos problemas (Teixeira, 1999).

\section{A epidemiologia no Sistema Único de Saúde}

A preocupação com a melhoria dos serviços do SU S e com a efeti vidade das intervenções sobre a situação de saúde tem motivado alguns autores a indagar sobre a incorporação da epidemiologia nas organizações de saúde em diferentes níveis do sistema de serviços de saúde e, especialmente, a sua utilização nas práticas de saúde (Schraiber, 1990; Paim, 1993; Teixeira, 1999; Drumond, 2001).

Ainda que a Constituição da República e a Lei Orgânica da Saúde (8.080/90), complementada pela lei $8.142 / 4$, indicassem muitos caminhos para a incorporação da epidemiologia no planejamento e gestão do SUS, elementos de inércia burocrática da saúde pública institucionalizada juntamente com a lógica inampsiana que dirigia o model o médico assistencial privatista dificultavam o desenvolvimento das práticas epidemiológicas. 


\section{A epidemiologia na implantação do SUS}

No início da década de 1990, a epidemiologia era confinada, no nível federal, à Fundação Nacional de Saúde (Funasa), instituída nos primeiros dias do Governo Collor, reunindo a Superintendência de Campanhas de Saúde Pública (Sucam) e a Fundação do Serviço Especial de Saúde Pública (FSESP). A criação do Centro Nacional de Epidemiologia (Cenepi), apesar de saudada por muitos epidemiologistas brasilei ros e não obstante certos esforços dos seus primeiros dirigentes no sentido de disseminar o saber epidemiológico entre as instituições de saúde (Teixeira, 1999), não reduziu a perspectiva de confinamento. No nível estadual, concentrava-se, por sua vez, nas ações de vigilância epidemiológica, sobretudo mediante os programas de imunização e controle de doenças implementados pelas estruturas próprias das secretarias de saúde, reforçando a dicotomia com as Coordenações Regionais da Funasa. E no âmbito municipal, a epidemiologia ten dia a ser residual, graças à própria insignificância que a lei 6.259/75 reservava para o município no então Sistema $\mathrm{N}$ acional de Vigilância Epidemiológica, além da fragilidade institucional das secretarias municipais de saúde. Portanto, os esforços para o uso da epidemiologia no planejamento e na gestão naquele período limitavam-se às proposições dos Planos Diretores de Epidemiologia (D rumond Jr., 2001) e às tentativas locais de desenvolvimento de model os assistenciais alternativos, com o apoio de universidades e de organismos de cooperação técnica (Teixeira, 2002).

M esmo assim, em seminário realizado pelo Cenepi, baseado em documento propondo a reorganização do Sistema de V i gilância Epidemiológica na perspectiva do SU S (Paim \& Teixeira, 1992), considerou-se que era necessário romper a dicotomia tradicional entre vigilância e assistência, possível a partir do aprofundamento da di scussão das propostas de Vigilância à Saúde e pela incorporação das ações de vigilância em todas as unidades de atenção à saúde, inclusive hospitais (Seminário N acional de Vigilância Epidemiológica, 1992).

Alguns elementos dessa mudança sobre os procedimentos técnicos, o uso de tecnologias e a integração de conhecimentos configuram, no caso brasileiro, o debate em torno dos mo- delos assistenciais iniciado nos anos 80 e disseminado na década de 1990. Já as mudanças nas formas de utilização dos recursos e de participação social tiveram proeminência com a edição da lei 8.142/90 e, especialmente, com a vigência das N OB/93 e N OB/96 (Brasil, 1993; 1996). Ao se examinar o contexto nacional, podem ser identificados componentes do arcabouço legal e normativo do SU S permeáveis à utilização da epidemiologia bem como certas relações desse saber com o planejamento e com os chamados model os tecnoassistenciais.

\section{Epidemiologia e descentralização}

A NOB/93, apesar de contribuir para o avanço da municipalização das ações e serviços de saúde, praticamente não contou com o parti cipação das instâncias respon sáveis pela epidemiologia no SUS, de modo que as suas orientações técnico-administrati vas deixam grandes vazios para o uso do saber epidemiológico como ferramenta de gestão. A adoção simultânea de políticas econômicas de ajuste estrutural, a implantação de propostas de Reforma do Estado, a restrição de recursos financeiros para o setor saúde e a opção por mecanismos de financiamento que privilegiam a lógica do mercado transformavam o setor público em prestador de serviços e o planejamento em mero instrumento de captação de recursos. A gestão tendia a se concentrar em aspectos administrativos, reproduzindo um estilo que privilegia a gerência contábil e 0 atendimento à demanda por serviços médico-ambulatoriais e hospitalares em detrimento das ações promocionais e de prevenção de agravos e danos à saúde col eti va, reforçando a iniqüidade no acesso aos serviços e desigualdades das condi ções de vida e saúde da população (Teixeira, 1999).

Assim, mesmo municípios sob a forma de gestão plena do sistema de saú de ap resentaram resultados modestos no que se refere ao uso da epidemiologia e à reorganização das práticas de saúde (H eimann et al., 1998), seja em relação à redefinição do objeto, dos meios de trabalho, do trabalho propriamente dito ou das relações técnicas e sociais sob as quais é realizado o trabalho.

Contudo, as possibilidades abertas com a implantação do SU S para a experimentação de modelos assistenciais; a produção teórica, epistemológica e metodológica sobre a epide- 
miologia na América Latina; o conjunto de proposições políticas e técnicas contidas nos Planos Diretores para o D esenvolvimento da Epidemiologia no Brasil I (1990-1994), II (1995-1999) elll (2000-2004) elaborados pela Associação Brasileira de Saúde Coletiva (Abrasco); e a implementação da política de munici palização das ações e serviços de saúde naúltima década compuseram um contexto no qual poderia favorecer a construção de uma "epidemiologia em serviços de saúde" (Drumond Jr, 2001). N esse particular, as promessas do Cenepi mediante o Vigisus (Brasil, 1998) chegaram a mobilizar secretarias de saúde interessadas em implementar o modelo da Vigilância da Saúde (Teixeira, 2002).

Ao levantar as formas predominantes dos instrumentos e modos de fazer epidemiologia que têm sido utilizados nos serviços de saúde no Brasil (Drumond Jr., 2001), o autor revelou usos criativos e inovadores da epidemiologia em serviços de saúde do SU S fornecendo indicações que já permitem vislumbrar certa reorganização das práticas sanitárias. Assim, os projetos $\mathrm{N}$ ascer em Curitiba, Risco Evolutivo e M ãe Curitibana são bons exemplos dos model os assistenciais correspondentes à oferta organizada (incluindo busca ativa), ao trabalho programático (ações programáticas de saúde) e à vigilância da saúde, implementados de forma complementar. Segundo o mencionado autor, os modelos de planejamento, tecnoassi stenciais e de organização dos serviços propostos no país, apesar de muitas identidades, têm visões diferenciadas sobre a ênfase e o uso da epidemiologia nos serviços de saúde (Drumond Jr., 2001). Por conseguinte, uma das grandes contribui ções desse estudo foi ressaltar as possibilidades e a criatividade de novos enfoques e temas realizando reflexões muito apropriadas sobre inovação institucional e incorporação tecnológica. N essa oportunidade, 0 autor critica o diagnóstico normativo em saúde pelo seu caráter ritualista, formalista e irresponsável em relação à gestão e à reorientação dos modos de inter venção em saúde. Assinala que a pretensa onipotência da epidemi ologia em definir necessidades e prioridades em saúde deve ser questionada e relativizada, ainda que possa ser considerada uma ferramenta de gran de utilidade, aténo diagnóstico estratégico de análise de situação de saúde. E assim o autor anuncia uma "epidemiologia do cotidiano e do atrevimento".

\section{Agenda de saúde e avaliação: uns passos adiante}

A partir da NOB/96 e, especialmente, com a expansão dos Programas de Agentes Comunitários de Saúde e de Saúde da Família (PACS/ PSF), favorecida pela adoção do Piso Assistencial Básico (PAB) em 1998, o M inistério da Saúde, através da Secretaria de Política de Saúde (SPS), vem introduzindo, progressivamente, certos dispositivos que tendem a valorizar a incorporação de práticas epidemiológicas na gestão do SUS, incluindo o apoio a estudos sobre aval iação de políticas e programas (Vieira da Silva et al., 2002).

N essa perspectiva, a aprovação pelo Conselho N acional de Saúde dos Eixos Prioritários de Intervenção para o Ano 2001 ilustra um passo importante para o envolvimento de estados e municípios na Agenda $\mathrm{N}$ acional de Saúde (Brasil, 2001). Entre as intervenções propostas destacam-se a redução da mortalidade infantil e materna e o controle de doenças e agravos prioritários, nos quais se incluem as "doenças da pobreza" (imunopreveníveis, transmitidas por vetores, diarréias, tuberculose, hanseníase, etc.), as doen ças crônico-degenerativas ( câncer, diabetes e hipertensão) e os chamados "novos desafios" (Aids e morbimortalidade por causas externas). As demais intervenções - 1) reorientação do modelo assistencial e descentralização; 2) melhoria da gestão, do acesso e da qualidade das ações e serviços de saúde; 3) desenvolvimento de recursos humanos do setor saúde; e4) qualificação do controle social - representam, também, espaços para o desenvolvimento de práticas epidemiológicas.

\section{Conjuntura pós-XI Conferência Nacional de Saúde: os tortuosos caminhos do SUS}

No caso da recente N orma O peracional de Assistência à Saúde (Brasil, 2001), em quepese a ausência no debate público da XI Conferência $N$ acional de Saúde, os riscos de recentralizacão da política setorial, o privilégio da hierarquização da assistência médico-hospitalar sob a denominação de "regionalização da assistência à saú de", bem como a ênfase na noção de economia de escala e a visão restrita de integralidade da aten ção (centrada na demanda espontânea e reduzida à idéi a de continuidade da assistência médica), caberia 
aproveitar a oportunidade da sua implementação para introduzir o saber epidemiológico nos processos de gestão (Teixeira, 2002).

Assim, a criação de "módulos assistenciais" e de "sistemas microrregionais de saúde" poderá ensejar um debate que venha a incorporar e articular práticas de promoção da saúde e prevenção de riscos e agravos, bem como a reorientação da assistência individual e coletiva (Teixeira, 2002). Enfim, a incorporação da proposta de Vigilância da Saúde ao planejamento municipal e regional poderá constituir uma alternativa de superação do viés economi cista da Programação Pactuada Integrada - PPI, basicamente um instrumento de racionalização da oferta de serviços pelas unidades de saúde, que não problematiza o conteúdo das práticas que são realizadas nem a sua adequação às necessi dades e problemas de saúde da população (Teixeira, 2002). A partir das propostas da autora, poderiam ser destacadas a redefinição das ações programáticas de saúde no âmbito das unidades básicas, a reestruturação dos estabelecimentos de saúde para assegurar a oferta organizada e programada das ações e serviços e a formulação de políticas públicas que tomem como referências básicas a promoção ea proteção da saúde (cidades sau dáveis, vigilância sanitária em defesa da saúde, ação intersetorial em saúde, empowerment, etc.).

A conjuntura em que a XI Conferência Nacional de Saúde defende o compromisso dos governos com políticas públicas integradas, com articulação intersetorial, capazes de assegurar as condições necessárias à produção, promoção e preservação da saúde éa mesma em que o Conselho $N$ acional de Saúde aprova a Política Nacional de Redução da M orbimortalidade por Acidentes e Violências e o M inistério da Saúde, implementa o Programa $\mathrm{Na}$ cional de Controle do Tabagismo (Barros, 2002), avança no Programa de Saúde da Família (PSF) - um dos espaços institucionais que em prega a prática epidemiológica - e busca, atualmente, formular uma política de promoção da saúde e uma política nacional de ciência \& tecnologia em saúde.

\section{0 agenciamento da epidemiologia: muitos passos atrás}

Asiniciativasmencionadas, ao lado daformulação e implementação da N OAS, ocorrem em um contexto marcado pela multiplicidade de eventos de caráter político-institucional que configuram uma trama complexa de deci sões acerca do processo de construção do SU S, nem sempre coerentesearticuladas(Teixeira, 2002). A autorasereferenessecaso à proposta decriação daAgência Federal dePrevenção eControledeD oenças (Apec), autarquia vinculada ao M inistério da Saúde, como "agência executiva", subsidiária da chamada Reforma do Estado.

Na realidade, sob o manto aparentedeReforma do Estado edemodernização da burocracia sanitá ria, toma corpo um processo de desmantelamento do organismo capaz de reduzir o paralelismo dos serviços eações de saúde, ou seja, o M inistério da Saúde, responsável pela gestão nacional do SUS(Paim, 2001). Esse "esquartejamento" do SUSseiniciacom a promulgação da Constituição quando assegurou quea saúdeélivreà iniciativa privada. M as avançou em 1998, com a criação da Agência N acional deVigilância Sanitária (Anvisa), apósuma avalanchededenúncias defalsificação demedicamentosenvolvendo empresasfarmacêuticas multinacionais derenome. $\mathrm{No}$ ano seguinte, tem continuidade com o estabelecimento daAgência N acional deSaúdeSuplementar, depoisdeum conjunto dedenúncias sobreos chamados "planos desaúde", vinculadosao Sistema deAssistência M édica Supletiva (SAMS). Em 2000 jásediscutiauma"agência devigilância epidemiológica", cuja proposta de M edida Provisóriafoi encaminhada pelo ministro daSaúde em julho do ano seguinte. No entanto, como dasoutrasvezes, foi necessáriaa existência demais denúnciasnamídia- no caso vinculadasà incompetência dosgovernos dianteda epidemia de dengue- para quea Presidência da República editasse maisuma M edidaProvisória(M P 33, 19/2/02) visando àtransformação daFunasa em A pec (Brasil, 2002).

Verifica-se, dessemodo, quea conjuntura pósXI Conferência N acional deSaúde, ao lado decertos avanços, traz sérios retrocessos no quediz respeito à incorporação das práticas epidemiológicas na gestão do SUS, conformeaavaliação daA brasco:

Esta M P ressuscita a lei $6.259 / 75$, queinstituiu o Sistema N acional deV Vigilância Epidemiológica, ignorando o papel do muniápio (...). Do mesmo modo atenta contra o princípio constitucional da integralidadeda atenção. Ao dispor sobreo Sistema Nacional deEpidemiologia confina o saber epidemiológico ea Epidemiologia, como disciplina científica, a um "sistema" paralelo, subordinado a uma agência para a execução das ações de prevenção e controle, justamente quando o país tenta avançar na adoção de modelosassistenciaismaisintegrais(...). . A presenta proposiçõesmaistruculentaseretrógradasqueasdesenvolvidas pela polícia sanitária do Brasil no início do século XX (...) A partir deuma vaga eindefinida 
"situação derisco", atenta contra os direitos dosindivíduos ecomunidades colocando- os no mesmo nível dosanimais! (Abrasco, 2002).

Sob o ponto devista da gestão das secretariasestaduaisemunicipais desaúde, a pulverização do comando federal sobreo SUS, além de configurar o risco defragmentação das orientações políticas, principalmente por separar o quesehavia tentado unir, isto é, a assistência médica (antigo I namps), a vigilância epidemiológica esanitária (antigo M S), representa um acréscimo de complexidadenas relações intergovernamentais com o nível federal (Teixeira, 2002). Ademais, osníveis estadual emunicipal do SU Spodem vir areproduzir, mecanicamente, à imagem esemelhança das estruturas federais, produzindo o reconfinamento da epidemiologia em certos guetos da burocracia sanitária. Como já sealertara há uma década, a organização sanitária brasileira já pagou um alto preço pelo mimetismo organizacional e pelo artificialismo das reformas administrativas(Paim \& Teixeira, 1992).

Diantedessequadro, 0 CNS aprovou a proposição de transformar a MP 33 em projeto de lei eo Congresso N acional rejeitou tal M P em 17/4/02. Lamentavelmente, o projeto delei quesubstituiu a M $\mathrm{P}$ ignorou olimpicamenteas críticas esugestõesapresentadasno V Congresso Brasileiro deEpidemiologiaeinsistena criação daApec, mantendo o "estado dequarentenafederal" no qual oscidadãosterão de sereportar, periodicamente, à "autoridadedeepidemiologia" (Abrasco, 2002).

Assim, a Reforma do Estado queo Governo tem implementado no setor saúde nos últimos anos, longedeatender aos pressupostos modernizantese democratizantes anunciados (Pereira \& Grau, 1999), caracteriza-sepela criação deagências como parte das reações espasmódicas dianteda crisesanitáriaedas denúncias da mídia. N essecontexto, a racionalidadetécnico-sanitáriaquea epidemiologia poderia proporcionar à gestão do sistema deserviços desaúdepraticamentedesapareceno processo decisório, predominando um conjunto deinteresses menores da burocracia associados aos das classes hegemônicas.

\section{Comentários finais}

Diante dos fatosacima examinadoseao se discutirem certas possibilidades de as práticas epidemiológicasserem recuperadas paraa gestão do SUS, épertinenteindagar qual epidemiologia estáno horizontedas propostas? A epidemiologia solidáriaà efetivação do SUS, através do acesso, qualidade ehumanização na atenção à saúde, com controle social, ou aquela que constrangeos cidadãos, submetendo-os à "autoridadedeepidemiologia" quepodeser um ministro da Saúde, um presidentedeAgência, um secretário deSaúde, um tecnoburocrata ou um inspetor dequarteirão?(Abrasco, 2002). A epidemiologiaquegera informação rel evante para os que sofrem os processos destrutivos da organização das cidadese, em últimaanálise, da lógica capitalista ou a epidemiologiaquemascaraa realidade, domesticae controla as populações deacordo com os interesses detecnoburocratas eem função dos projetospolítico-ideológicos das classes dirigentes? U ma epidemiologiados "decima" parareproduzir seus privilé gioseexclusão social ou dos "debaixo" para produzir informação epoder em busca da eqüidadeeda efetividade?

Portanto, aepidemiologia, como disciplinacientífica ou como ferramenta degestão, não éinocente, neutra, nem paira sobre os interesses declasses eos respectivos projetos políticos eideológicos. Como prática social, não se encontra livre das determinações quea estrutura social faz incidir sobreas práticas desaúde. Dianteda crise da disciplina, distintas propostastêm sido formuladas por epidemiologistas epensadores da medicina social eda saúdecoletivalatino-americanataiscomo: a) recuperar as experiênciasrelevantesacumuladasnahistóriada epidemiologia e de outras disciplinas queestudam a saúdeeseus determinantes; $b$ ) redirecionar o desenvolvimento teórico, metodológico eoperacional da disciplina; c) deslocar o atual modus operandi da prática científica da epidemiologia para temas de prevenção epara o desenvolvimento denovas bases éticas, coerentes com seus compromissos sociaise históricos (Barreto, 1998); d) proceder auma análise crítico-epistemológica decategorias centraiscomo "causalidade", "determinação", "risco", "exposição"; e) promover uma discussão ampla sobremetodologia(Breilh, 2002).

Seaepidemiologia for pensada paraalém dasua dimensão técnica, outrosdesafiosapresentar-seiam dianteda práxis. N essa perspectiva, teria deser uma testemunha crítica dos processos destrutivos para a vidaqueserealizam na sociedade, uma ferramenta demonitoramento da qualidadedevidaeda saúde, um instrumento de "empoderamento" da população euma arma deplanificação estratégica eparticipativa(Breilh, 1998).

Portanto, revisar criticamente o paradigma científico dominantepodeser uma via deanalisar certas possibilidades detransição paradigmática no campo da saúde coletiva, dianteda complexidade do objeto da epidemiologia. N esse caso, caberia aproximar a ciência da sociedade eseus problemas, ampliando sua capacidadedeproduzir conhecimen- 
to crítico epropositivo. Conhecimento ético, emandpador, solidário e democrático (Drumond, 2001). Evidentementequenuma estrutura social quenega taisvalores, a epidemiologia enfrenta sérias contradições: na sua tensão entre disciplina científica e campo profissional, a epidemiologia traz à tona, para os seus praticantes, independentementede onde estejam situados, os desafios da dialética entreo sonhar e o fazer, entre a utopia ea realidade, entrea técnica ea política (Barreto, 1998).

Ainda que muitas questões relevantes na atualidade sejam insuficientemente consideradas pela epidemiologia hegemônica, caberia destacar certos "objetivos essenciais" deum saber quetomepartido pela vida e pela emancipação dos seres humanos. U ma epidemiologia que fundamenteas ações em saúdecoletiva, inspirada nos seus compromissos democráticos desde as lutas históricas do movimento sanitário contra o autoritarismo. Uma epidemiologiaquepossa garantir o conhecimento do processo saúde-doença na realidade complexa e concreta; reconhecer eabordar suas relações em diferentes níveis da realidade buscando se integrar com as vi sões de diferentes disci plinas e profissionais para orientar intervenções; econtribuir na redução do sofrimento humano, das iniqüi dades sociais detectadas eno movimento em defesa da vida (Drumond Jr., 2001). Trata-se, enfim, deconstruir coletivamente as bases deuma epidemiologia contra-hegemônica que examineo movimento geral da sociedadee suas relações com o modo de vida dos grupos sociais e com o estilo de vida das pessoas, identificando processos críticos deexposição ou deimposição (Breilh, 2002), conformeas palavrasdo autor:

H ablar de praxis epidemi ológica a comienzos del novo milenio no es lo mismo que hacerlo cu- ando fundábamos el movimiento de la salud colectiva en la década delos setenta etrabajábamos en las primeras rupturas (...). No somos los mismos pero somos i guales. No somos los mismos porquenuestra praxis há experimentado cambios y acumulaciones deci si vas, nuestras propuestas se han enriquecido, nuestras ideas han crecido en amplitud y estensión. Pero somosiguales, porque seguimos siendo humanistas, en el más profundo y marxista sentido dela palabra, el sentido deforjar identidad e recrear utopía emanci padora (Breilh, 2002).

U ma epidemiologia que contribua na constituição de sujeitos sociais comprometidos com uma prática sanitária voltada para a generosidade, a solidariedade e a ética na luta pela saúde e qualidade de vida, representa uma aposta na planificação e gestão de um sistema de saúde que se preten de efetivo, democrático, humanizado e equânime. M ais que uma aposta, tais processos de construção contra-hegemônica implicam una articulación organizativa entre sujetos como condición previa a la transformación de las prácticas, tal como lo queria Gramsci, al generar un nuovo pensami ento que no sólo cuestiona los saberes tradicionales sino que pude constituirse en el liderazgo para proponer uma nuova manera de hacer las cosas (Testa, 1997). Portanto, a explicitação, a disseminação e a apropriação desses val ores pelas classes subalternas e seus intelectuais orgânicos poderá favorecer a construção de identidades capazes de influir na mobilização de subjetividades e vontades políticas para a concretização de práticas epidemiológicas e de gestão comprometidas com os princípios e diretrizes originais do SUS.

\section{Referências bibliográficas}

Abrasco 2002. Editorial. Outra emenda pior que o soneto. Apec - Novas Ameaças ao Processo de Descentralização da Saúde. Boletim A brasco 84:23.

Barata RB 1998. Epidemiologia e saber científico. Revista Brasileira de Epidemiologia 1(3):14-27.

Barreto ML 1998. Por uma epidemiologia da saúde coletiva. Revista Brasileira de Epidemiologia 1(3):104-122.

Barros E 2002. A vanços e desafios para o SU S face às principais recomendações da XI Conferência $\mathrm{Na}$ cional de Saúde. (Texto elaborado para subsidiar 0 Conselho Nacional de Saúde).

Brasil. M inistério da Saúde 1993. N orma O peracional Básica SU S 01/1993. Informe Epidemiológico do SUS, número especial:49-73.

Brasil. M inistério da Saúde 1996. N orma O peracional 
Básica SU S 01/96. D OU 6/11/96.

Brasil. M inistério da Saúde/Cenepi 1998. Vigisus S. N. $\mathrm{T}$.

Brasil. M inistério da Saúde 2001. Portaria № 393. D O U 30/03/01.

Brasil. M inistério da Saúde 2001. Regionalização da assistência à saúde: aprofundando a descentralização com equidade no acesso ( $\mathrm{N}$ orma O peracional da Assistência à Saúde. N O AS/SU S 01/01. Portaria M S/GM No 95, de 26 de janeiro de 2001) Brasília, DF, 114p.

Brasil. Presidência da República/Casa Civil 2002. M edida Provisória № 33. DOU 19/2/02.

Breilh J 1998. La sociedad, el debate de la modernidad y la nueva epidemiologia. Revista Brasileira de Epidemiologia 1(3):207-233.

Breilh J 2002. N ueva epidemiologia: construcción intercultural de otro paradigma de la ciencia. Tese de doutorado. Instituto de Saúde Coletiva, Salvador, Brasil. 226p.

CDC 1992. Proceedings of the 1992 International Symposium on Public Health Surveillance. M M WR 41/Supplement, 218p.

Donnângelo MCF 1976. Saúde e sociedade. Duas Cidades, São Paulo.

Dussault G 1995. La epidemiologia y la gestión de los servicios de salud.. Boletin Epidemiológico OPS 16(2):1-5.

Drumond Jr, M 2001. Epidemiologia e saúde pública: reflexões sobre os usos da epidemiologia nos serviços do Sistema Ú nico de Saúde em nível municipal. Curso de Pós-Graduação do Departamento de M edicina Preventiva e Social da Faculdade de Ciências M édicas da Universidade Estadual de Campinas.

H eimann LS et al. 1998. A descentralização do sistema de saúde no Brasil - uma proposta de investigação sobre o impacto de políticas. Relatório Final. Instituto de Saúde, São Paulo.

$M$ endes- $G$ onçalves RB 1994. Tecnologia e organização das práticas de saúde: características tecnológicas do processo de trabalho da rede estadual de centros de saúde de São Paulo. Hucitec-Abrasco, São PauloRio deJaneiro.

Merhy EE 1997. Em busca do tempo perdido: a micropolítica do trabal ho vivo em saúde, pp. 71112. In EE M erhy \& R Onocko (org.). Agir em saúde: um desafio para o público. Hucitec-Lugar Editorial, São Paulo-Buenos Aires.

Morris JN 1975. U ses of epidemiology. 3a ed. Churchill Livingstone, Edimburgo.

M ota P 1992. Gestão contemporânea: a ciência e a arte de ser dirigente. Record, Rio de Janeiro.

OPS 1984. Documento del seminario sobre U sos y Perspectivas de la Epidemiologia, Buenos Aires 7-10 N oviembre. Boletin Epidemiológico 5(1):1-4.

OPS 1988. El desafio de la epidemiologia, problemas y lecturas selecionadas. Publicación Científica 505:881-889.

OPS 1991. Vigilancia de la situación de salud según condiciones de vida. Boletin Epidemiológico
12(3):7-10.

OPS/OM S 1992. D esarrollo y fortalecimiento de los sistemas locales de salud: la administración estratégica. Washington, D.C. 160p.

Paim JS 1993. A reorganização das práticas de saúde em distritos sanitários, pp. 187-220. In EV Mendes (org.). Distrito Sanitário: o processo social de mudança das práticas sanitárias do Sistema Ú nico de Saúde. Hucitec-Abrasco, São Paulo-Rio de Janeiro.

Paim JS 1999. Por um planejamento das práticas de saúde. Ciência \& Saúde Coletiva 4(2):243-24.

Paim JS 2001. Transición paradigmática y desarrollo curricular en salud pública, pp. 85-112. In OPS. Educación en salud pública: nuevas perspectivas para las A méricas. Washington, D.C. OPS.

Paim JS 2002. Saúde, política e reforma sanitária. CEPS/ ISC, Salvador.

Paim JS \& Teixeira M GLC 1992. Reorganização do Sistema de Vigilância Epidemiológica na perspectiva do Sistema Ú nico de Saúde. Informe Epidemiológico do SUS 5:27-57.

Pereira LCB \& Grau NC (org.) 1999. 0 público não-estatal na reforma do Estado. Editora Fundação Getúlio Vargas, Rio de Janeiro.

Rivera FJU 1995. A gir comunicativo e planejamento social (uma crítica ao enfoque estratégico). Fiocruz, Rio de Janeiro.

Sá M C 1993. Planejamento Estratégico em Saúde: problemas conceituais e metodológicos. Dissertação de mestrado, Escola Nacional de Saúde Pública, Fiocruz, Rio de Janeiro.

Schraiber LB (org.) 1990. Programação em saúde, hoje. Hucitec, São Paulo.

Schraiber LB et al. 1999. Planejamento, gestão, e avaliação em saúde: identificando problemas. Ciência \& Saúde Coletiva 4(2):221-242.

Seminário N acional deVigilância Epidemiológica 1992. Relatório final. Informe Epidemiológico do SU S 5:524.

Teixeira CF 1996. Epidemiologia e planejamento em saúde: contribuição ao estudo da prática epidemiológica no Brasil 1990-1995. Tese de doutoramento, ISC/UFBA. 300p.

Teixeira CF 1999. Epidemiologia e planejamento de saúde. Ciência \& Saúde Coletiva 4(2):287-303.

Teixeira C (org.) 2002. Promoção e vigilância da saúde. CEPS/ISC, Salvador.

Testa M 1992. Pensar em saúde. Artes M édicas-A brasco, Porto Alegre-Rio de Janeiro.

Testa M 1997. Saber en salud. La construcción del conocimiento. Lugar Editorial, Buenos Aires.

Tigre C et al. 1990. La práctica epidemiológica en los sistemas de servivios de salud. Educ. M éd. Salud 24(3): 306-320.

Vieira da Silva LM , H artz ZM A, Costa M CN, Oliveira $A B$, Biscarde $P \& P a z B M$ 2002. Avaliação dos efeitos da descentralização em relação às condições traçadoras da aten ção à saúde. U niversidade Federal da Bahia/Instituto de Saúde Coletiva/Centro Colaborador do Ministério da Saúde, Relatório Preliminar.

Artigo apresentado em 20/10/2002

A provado em 30/11/2002

Versão final apresentada em 10/12/002 